\title{
Combination Effects of Herbs in a Multi-herbal Formula: Expression of Juzen-taiho-to's Immuno-modulatory Activity on the Intestinal Immune System
}

\author{
Hiroaki Kiyohara ${ }^{1,2}$, Tsukasa Matsumoto ${ }^{1,2}$ and Haruki Yamada ${ }^{1,2}$ \\ ${ }^{1}$ Kitasato Institute for Life Sciences, Kitasato University, Tokyo and ${ }^{2}$ Oriental Medicine Research Center, The Kitasato \\ Institute, Tokyo, Japan
}

\begin{abstract}
Herbal formulas of traditional Japanese (Kampo), Chinese and Korean medicines usually comprise multiple herbs in a single formula. These medicines are expected to show their clinical effects by chemical, pharmacological and pharmaceutical combination effects of multi-herbs. However, little effort has been made so far to scientifically clarify the nature of such combination effects. Interestingly, for example, though a Kampo medicine Juzen-taiho-to (Shi-Quan-Da-Bu-Tang in Chinese) stimulates the immune functions of Peyer's patch cells, none of its single component herbs shows such activity. We thus examined the combination effect of herbs in the Juzen-taiho-to formula for the expression of its immuno-stimulating activity. Juzen-taiho-to, a composite formula of 10 herbs, has been generally considered to comprise two kinds of basic formula, each of which consists of four different herbs in addition to two others. The combinations of herbs based on these two basic formulas were evaluated for their stimulating activities on cytokine production from murine Peyer's patch cells both in vitro and ex vivo. Combined decoction of six among 10 herbs in Juzen-taiho-to is crucial for the expression of its stimulating activity on Peyer's patch cells. 3D-HPLC analysis of the ingredients in the fractions from the combined decoctions indicated that, in addition to quantitative changes of ingredients, alterations occur in their chemical composition by decoction of different herbs. The stimulating activity of Juzen-taiho-to on Peyer's patch cells results from the combination effect of its six essential component herbs. This combination effect is based on physicochemical interactions among the ingredients of the component herbs.
\end{abstract}

Keywords: combination effect - lignin-carbohydrate complexes - Peyer's patch cells

\section{Introduction}

A number of herbal formulas of traditional Japanese medicines (Kampo), and Chinese and Korean ones as well, are characterized by the use of mixtures of several herbs (multiherbs) in a single formula. These multi-herb formulas are usually prepared in various dosage forms such as decocted extracts, pills, powders, tablets etc. in a traditional way. Therefore, particularly in the case of decocted extracts with boiling water, one has to expect the possibility that some chemical interactions take place among natural constituents existing in the component herbs of the formula during decoction. Decoction may change the extraction rates of the active ingredients and/or produce new artificial substances, which

For reprints and all correspondence: Haruki Yamada, Kitasato Institute for Life Sciences, Kitasato University, Minato-ku, Tokyo 108-8641, Japan.

E-mail: yamada@lisci.kitasato-u.ac.jp may then exhibit new pharmacological activities. These types of pharmaceutical actions, derived from the combination of multiple herbs in a formula, are called 'chemical combination effects'. Meanwhile, it has also been postulated that in multiherbal formulas, the pharmacological activities of one single herb is either potentiated or prolonged, and/or its adverse effects reduced, due to synergistic or antagonistic effects, by addition of other herbs (1). These complex phenomena are supposed to emerge as a number of different active ingredients interact with each other, acting on different target systems in the body. It is also possible that certain pharmacokinetic situations such as concentrations in blood and metabolism of ingredients are altered in the presence of other ingredients which may affect drug transporters and drug metabolizing enzymes. These types of pharmacological action are called either "pharmacological combination effects' or 'pharmaceutical combination effects'. However, because of the sheer complexity of the phenomena, not many 
Table 1. Component herbs of Juzen-taiho-to and its basic formulations

\begin{tabular}{|c|c|c|c|c|c|c|c|c|c|}
\hline \multirow[t]{2}{*}{ Component herb } & \multirow[t]{2}{*}{ Juzen-taiho-to } & \multicolumn{3}{|c|}{ SMT } & \multicolumn{4}{|c|}{ SKT } & \multirow[t]{2}{*}{ SMT and SKT } \\
\hline & & alone & with ASR & with $\mathrm{CC}$ & alone & with ASR & with $\mathrm{CC}$ & with ASR and CC & \\
\hline Rehmanniae Radix (3 g) & * & $*$ & * & $*$ & & & & & $*$ \\
\hline Cnidii Rhizoma (3 g) & $*$ & $*$ & $*$ & $*$ & & & & & $*$ \\
\hline Angelicae Radix (3 g) & $*$ & $*$ & * & $*$ & & & & & $*$ \\
\hline Atractylodis lanceae Rhizoma (3 g) & * & & & & * & * & * & $*$ & $*$ \\
\hline Poria (3 g) & $*$ & & & & * & * & $*$ & * & $*$ \\
\hline Glycyrrhizae Radix (3 g) & * & & & & * & $*$ & $*$ & $*$ & $*$ \\
\hline Astragali Radix (ASR, $1.5 \mathrm{~g}$ ) & * & & * & & & * & & $*$ & \\
\hline Cinnamomi Cortex (CC, $3 \mathrm{~g})$ & * & & & * & & & $*$ & * & \\
\hline $\begin{array}{l}\text { Intestinal immune system } \\
\text { modulating activity (in vitro) }\end{array}$ & $\dagger$ & $\ddagger$ & $\ddagger$ & $\ddagger$ & $\ddagger$ & $\S$ & $\ddagger$ & $\dagger$ & ‡ \\
\hline
\end{tabular}

Asterisks indicate presence of herb in the formulation listed at the top of each column.

†Full activity.

No activity.

${ }^{\S}$ Statistically significant activity.

researchers have tackled this problem by performing actual experiments to scientifically elucidate these interesting effects.

Juzen-taiho-to (Shi-Quan-Da-Bu-Tang in the Chinese pronunciation) is one famous formula in Kampo, Chinese and Korean medicines, which comprises 10 different kinds of single herbs. Juzen-taiho-to has been clinically used in Japan for the treatment of patients recovering from surgery or suffering from chronic disease, to promote improvement of their debilitated general condition. By pharmacological evaluation using a pharmaceutically homogeneous preparation (TJ-48) of Juzen-taiho-to, it has been found that the herbal formula not only affects the systemic immune functions of $\mathrm{T}$ and $\mathrm{B}$ cells, macrophages and NK cells, but also those of the hematopoietic and intestinal immune systems (2-10). It has been reported that several kinds of macromolecular polysaccharides, especially lignin-carbohydrate complexes, and numerous kinds of low molecular weight components are its active ingredients (9-15). Some of these active ingredients have also been identified in some of its single herb components $(11,12)$. Although the lignin-carbohydrate complexes obtained from TJ-48 could show a potent modulatory activity on the intestinal immune system, lignin-carbohydrate complexes from any of the 10 single herbs in TJ-48 failed to show such activity, indicating the existence of certain combination effects among multiple herbs in the formula.

We therefore aimed in the present study to evaluate the potential combination effects of herbs in the Juzen-taiho-to formula in its modulatory activity on the intestinal immune system.

\section{Subjects and Methods}

\section{Materials}

Spray-dried extract preparation (TJ-48) of Juzen-taiho-to was kindly supplied by Tsumura \& Co. (Tokyo, Japan). TJ-48 was prepared as described previously (14). Astragali Radix (roots of Astragalus membranaceus Bunge), Rehmanniae Radix (roots of Rehmania glutinosa Libosch var. purpurea Makino), Paeoniae Radix (rhizomes of Paeonia lactiflora Pall), Cnidii Rhizoma (rhizomes of Cnidium officinale Makino), Atractylodis Lanceae Rhizoma (rhizomes of Atractylodes lancea DC), Ginseng Radix (roots of Panax ginseng C.A.Meyer), Poria (fungi of Poria cocos Wolf) and Glycyrrhizae Radix (roots of Glycyrrhiza uralensis Fisch et DC) were purchased from Uchida-Wakan-Yaku Co. (Tokyo, Japan) and Cinnamomi Cortex (barks of Cinnamomum cassia Blume) and Angelicae Radix (roots of Angelica acutiloba Kitagawa) were obtained from Tochimoto-Tenkaido (Osaka, Japan).

\section{Preparation of Lignin-Carbohydrate Complexes Containing Fraction (F-3) From TJ-48 and the Corresponding Fractions (F-3-related Fractions) From Each Single Herb}

The lignin-carbohydrate-complex-containing fraction, which we call ' $\mathrm{F}-3$ ', was prepared from TJ-48 according to the procedure described previously (14). The corresponding fractions (F-3-related fractions) were also obtained from each single herb and the mixture of single herbs was obtained in a similar manner. Briefly, $10 \mathrm{~g}$ of each component herb was decocted with $100 \mathrm{ml}$ of water to half volume, and the residual materials were re-decocted by the same procedure. After the combined extract was concentrated to $50 \mathrm{ml}$ by evaporation, $4 \times$ vol of $\mathrm{EtOH}$ was added to the solution, and the 
resulting precipitate was removed. The resulting supernatant was evaporated to dryness, and hydrophobic low-molecularweight ingredients were removed by refluxing with $\mathrm{MeOH}$ $(100 \mathrm{ml}, 1 \mathrm{~h})$. The $\mathrm{MeOH}$-insoluble materials were dialyzed by cellulose dialysis tube (molecular weight cut off: 12000 14000 ) against distilled water for 4 days. The dialyzable portion was recovered and evaporated to obtain water-soluble and dialyzable fraction as F-3-related fractions of the component herbs.

The two kinds of basic formula-Shikunshi-to (SKT) (SiJun-Zi-Tang in Chinese pronunciation) and Shimotsu-to (SMT) (Si-Wu-Tang in Chinese pronunciation) - and the corresponding combinations of single component herbs of Juzen-taiho-to were prepared as shown in Table 1. These formulas and combinations were decocted with $600 \mathrm{ml}$ of water to half volume, and the residual materials were re-decocted by the same procedure. The combination of two single herbs, Astragali Radix (ASR) (3 g) and Cinnamomi Cortex (CC) $(3 \mathrm{~g})$ was also decocted in the same manner. The resulting extracts were concentrated to $50 \mathrm{ml}$, and the water-soluble and dialyzable fractions from these decoctions were prepared as F-3-related fractions by the same procedure as described above, using $\mathrm{MeOH}$ extraction, EtOH precipitation and dialysis.

\section{Measurement of Intestinal Immune System Modulating Activity}

In vitro and in vivo activities were measured as described previously $(10,15)$. For in vivo experiments, suspensions of Peyer's patch cells in RPMI-1640 medium (Gibco, Grand Island, NY) supplemented with $5 \%$ fetal bovine serum (RPMI 1640-FBS) were prepared at day 8 from the small intestine of $\mathrm{C} 3 \mathrm{H} / \mathrm{HeJ}$ mice (female, 6-8 weeks old, SLC, Japan), who had been orally administered with F-3-related fractions $(150 \mathrm{mg} / \mathrm{kg} /$ day) or distilled water (control) for one week. Aliquots $(200 \mu \mathrm{l})$ of the cell suspension $\left(1-2 \times 10^{6}\right.$ cells/ml in RPMI 1640-FBS) of Peyer's patch cells were cultured in 96-well flat bottom microtiter plates for 6 days at $37^{\circ} \mathrm{C}$ in a humidified atmosphere of $5 \% \mathrm{CO}_{2}-95 \%$ air.

For in vitro experiments, $20 \mu \mathrm{l}$ of aqueous solutions of samples or distilled water were cultured for 6 days with $180 \mu \mathrm{l}$ of cell suspension of Peyer's patch cells, prepared from the small intestine of $\mathrm{C} 3 \mathrm{H} / \mathrm{HeJ}$ mice. The resulting culture supernatants $(50 \mu \mathrm{l})$ obtained by in vitro and in vivo experiments were further cultured with bone marrow cell suspension $\left(2.5-5.0 \times 10^{5}\right.$ cells $\left./ \mathrm{ml}\right)$, which were prepared from $\mathrm{C} 3 \mathrm{H} / \mathrm{HeJ}$ mice, in a humidified atmosphere of $5 \% \mathrm{CO}_{2-}$ $95 \%$ air for 6 days. At 5-30 h prior to culture termination, $20 \mu \mathrm{l}$ of Alamar Blue ${ }^{\mathrm{TM}}$ solution (Alamar Bio-Sciences Inc., Sacramento, CA) was added to each well, and the cells were then continuously cultured. The fluorescence intensity was measured by Fluoroscan II (Labosystems) at an excitation wavelength of $544 \mathrm{~nm}$ and an emission wavelength of 590 $\mathrm{nm}$. The modulatory activity on the intestinal immune system was expressed as fluorescence intensity compared to that of control. In vitro activity of each sample was obtained from four experiments in all case, and in vivo activity from 36 experiments. The results of fluorescence intensity are varied depending on the conditions of Peyer's patch cells and bone marrow cells in this assay system, and are comparable among the results in the same culture plate.

\section{Statistics}

All results are expressed as the mean \pm S.E. The differences between the control and the treatments in these experiments were tested for statistical significance by Student's $t$-test (in vitro) and Welch's $t$-test (in vivo). The differences between the treatments were tested by Scheffe test (in vitro). A value of $P<0.05$ was considered to indicate statistical significance.

\section{Analytical Methods}

Total carbohydrate and uronic acid were determined by phenol- $\mathrm{H}_{2} \mathrm{SO}_{4}$ and $m$-hydroxybiphenyl methods, respectively $(16,17)$. Lignin content was analyzed by Bradford's method with Bio-Rad dye (Bio-Rad) (18), because the color reagent in this method cross-reacts with standard lignin $(\mathrm{H}$. Kiyohara, unpublished data). Component sugars of the samples were analyzed as trimethylsilyl methylglycoside derivatives by GLC as described previously (15).

F-3-related fraction prepared from SKT and SKT with ASR and CC were fractionated on Sephadex G-25 equilibrated with $\mathrm{H}_{2} \mathrm{O}$ and the eluted materials were analyzed for carbohydrate, uronic acid and lignin-like molecules by the phenol- $\mathrm{H}_{2} \mathrm{SO}_{4}$ method, the $m$-hydroxybiphenyl method and measurement of UV absorption at $280 \mathrm{~nm}$. Three dimensional (3D-)HPLC analyses were performed on Agilent 1100 Series HPLC system equipped with photodiodarray detector and TSK gel-ODS 80Ts column $(4.6 \times 250 \mathrm{~mm}$, Toso Co. Ltd). The 3D-HPLC conditions were as follows: flow rate at $0.8 \mathrm{ml} / \mathrm{min} ; 10 \mathrm{mM} \mathrm{H}_{3} \mathrm{PO}_{4}-\mathrm{CH}_{3} \mathrm{CN}=95: 5 \rightarrow 5: 95$ (60 min, linear gradient); monitored from 200 to $600 \mathrm{~nm}$.

\section{Results}

The fractions (F-3) containing lignin-carbohydrate complexes, which were prepared from Juzen-taiho-to (TJ-48), and F-3-related fractions from hot water extracts of each of the 10 single herbs of TJ-48, were compared for their modulatory activity on the intestinal immune system. Although F-3 obtained from TJ-48 exhibited a potent activity, none of the F-3-related fractions from each single component herb had such activity (Fig. 1). When F-3-related fractions of the 10 single herbs were mixed in equal amounts, or in ratios according to their yields, and were measured for activity, these mixed F-3-related fractions also failed to express any activity (Fig. 2A). These results clearly indicate that the combined decoction of over two kinds of single herbs in the formula of Juzen-taiho-to is necessary for obtaining F-3 possessing activity. To further analyze this interesting phenomenon, identification of the essential combination is necessary. How- 


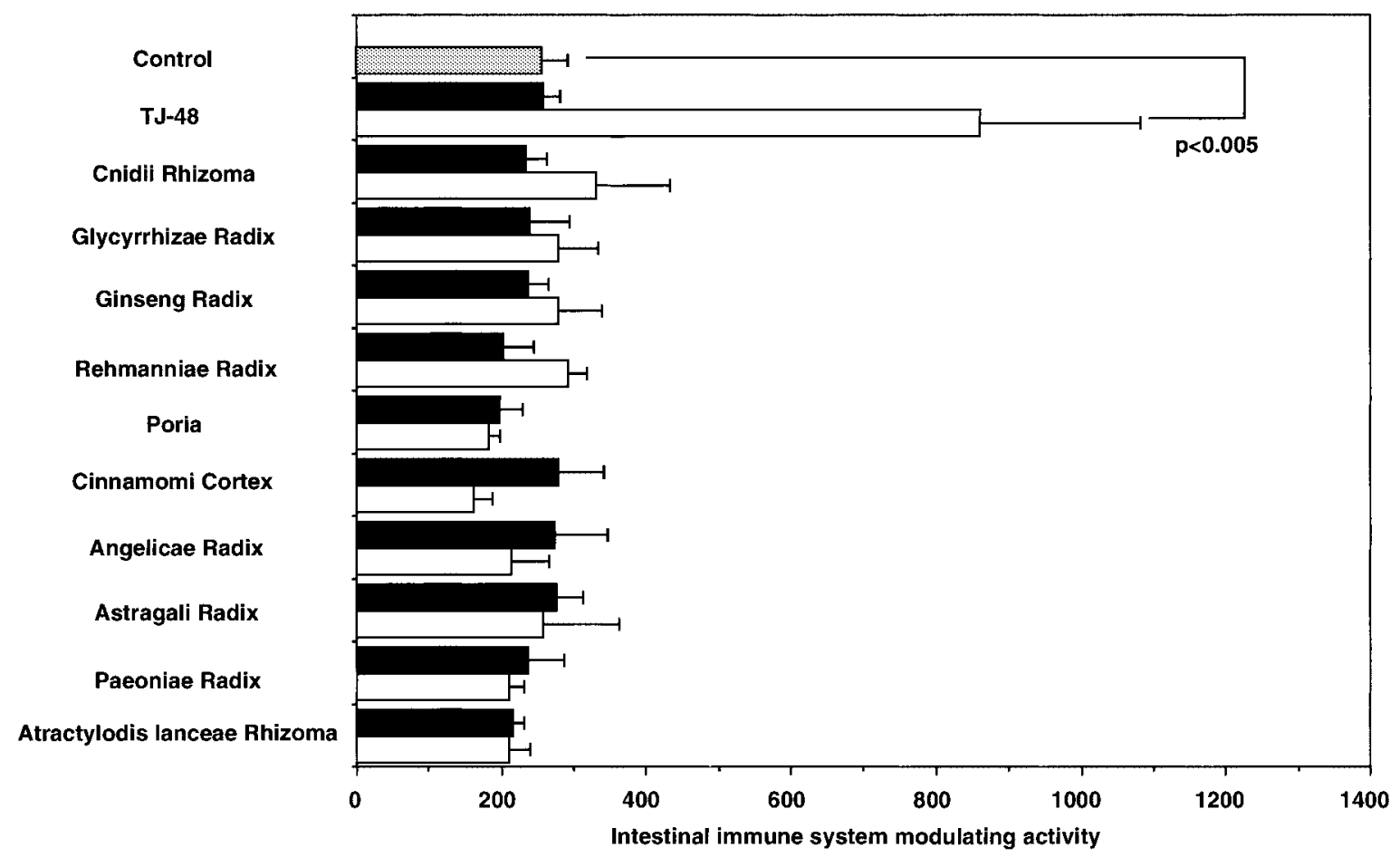

Figure 1. Intestinal immune system modulating activity of lignin-carbohydrate complexes containing fractions (F-3) prepared from Juzen-taiho-to (TJ-48) and F-3-related fractions from component herbs. Filled squares, $10 \mu \mathrm{g} / \mathrm{ml}$; open squares, $100 \mu \mathrm{g} / \mathrm{ml}$.
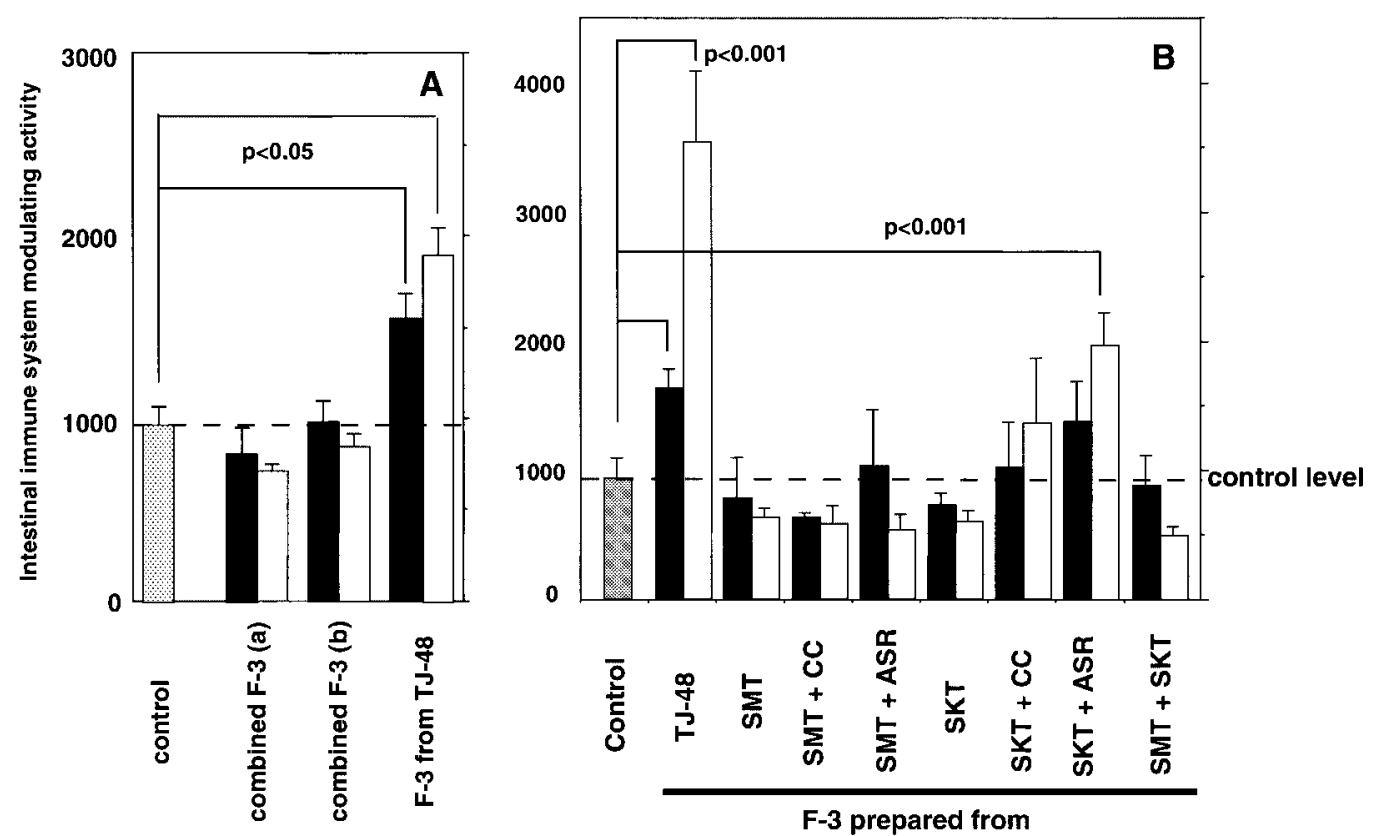

Figure 2. (A) Intestinal immune system modulating activity of combined F-3-related fractions, which were prepared by mixing of F-3-related fractions obtained from each of the 10 component herbs in equal ratios (a) and in ratios according to their yields (b). (B) Intestinal immune system modulating activity of various F-3-related fractions prepared from decoctions of SMT or SKT with or without ASR or CC, and from the decoction of the combination of SMT and SKT. Filled squares, $10 \mu \mathrm{g} / \mathrm{ml}$; open squares, $100 \mu \mathrm{g} / \mathrm{ml}$.

ever, in identifying the essential combination of single herbs for the expression of activity, it would be extremely inefficient if F-3-related fractions were prepared from random combinations of the 10 single herbs: numerous kinds of over 100 combinations have to be evaluated.
The formula of Juzen-taiho-to is generally recognized in the theory of Oriental herbal medicine to comprise two kinds of basic formulas, SMT (consisting of four single herbs: Rehmanniae Radix, Paeoniae Radix, Cnidii Rhizoma and Angelicae Radix) and SKT (consisting of four single herbs: 


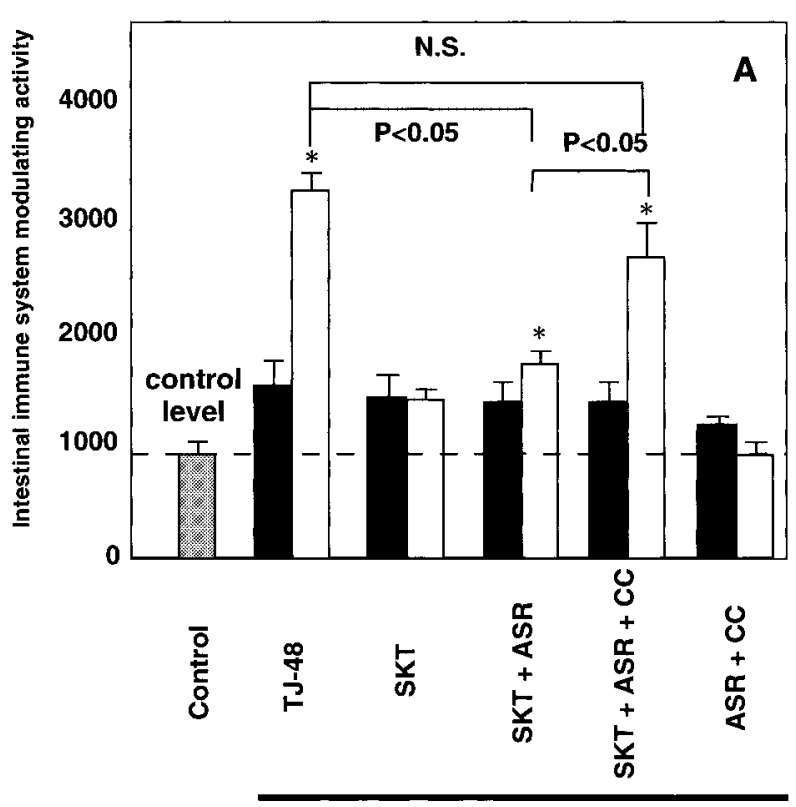

F-3 prepared from

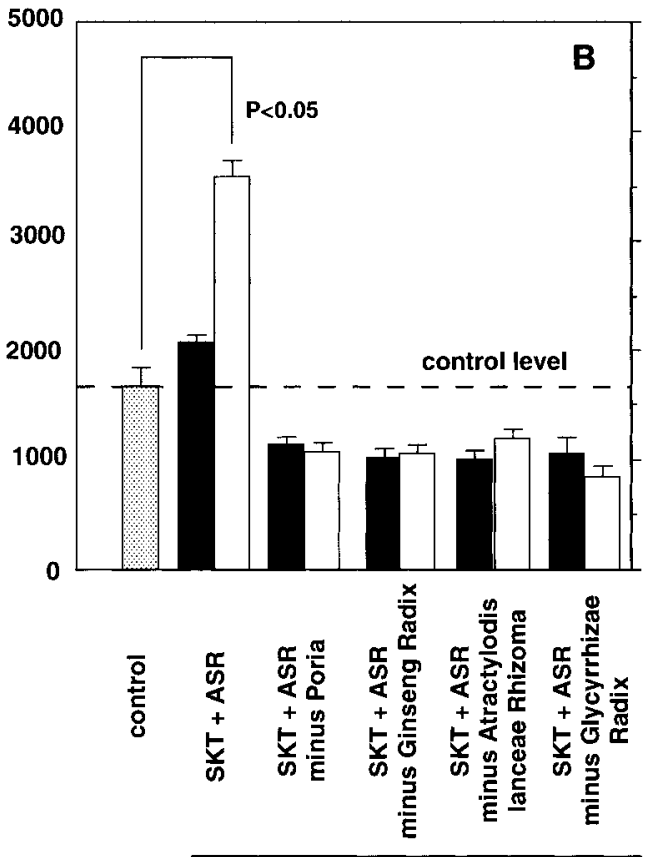

F-3 prepared from

Figure 3. (A) Intestinal immune system modulating activity of various F-3-related fractions prepared from decoctions of SKT with ASR alone or with ASR and CC combined, and from decoction of the combination of ASR and CC. (B) Effect of omitting each component herb from the combination of SKT with ASR on intestinal immune system modulating activity of their F-3-related fractions. Filled squares, $10 \mu \mathrm{g} / \mathrm{ml}$; open squares, $100 \mu \mathrm{g} / \mathrm{ml}$. ${ }^{*} P<0.05 \mathrm{versus}$ control. N.S., not significant.

Atractylodis Lanceae Rhizoma, Ginseng Radix, Poria and Glycyrrhizae Radix) in addition to ASR and CC (Table 1). Therefore, we selected multi-herbs according to the formulas of SMT, SKT or SMT combined with SKT, each of which were decocted, and F-3-related fractions were prepared to measure activity. We found that none of the F-3-related fractions showed activity, however, which indicated that the multi-herbs within eight kinds of single herb based on the formulas of SMT and SKT do not contribute to the expression of activity (Fig. 2B, Table 1). When other single herbs, ASR or $\mathrm{CC}$, were also combined with the SMT formula, F-3related fractions prepared from the combined decoctions of herbs in these combinations did not show any intestinal immune system modulating activities either (Fig. 2B, Table 1). However, although F-3-related fraction obtained from the combined decoction of herbs in SKT with CC showed no activity, the F-3-related fraction prepared from SKT with ASR did exhibit a statistically significant activity (Fig. 2B, Table 1). It was also discovered that the combined decoction of CC with the five single herbs in SKT with ASR significantly increased the activity of its F-3-related fraction to the same level as F-3 from TJ-48 (Fig. 3A, Table 1). In contrast, the F-3-related fraction prepared from the combined decoction of two kinds of single herbs, ASR and CC, had no such activity (Fig. 3A).

We then orally administered F-3-related fractions (150 $\mathrm{mg} / \mathrm{kg}$ /day), prepared from the combined decoction of SKT and from that of SKT with ASR and CC, to $\mathrm{C} 3 \mathrm{H} / \mathrm{HeJ}$ mice for 1 week. It was discovered that the F-3-related fraction from the combination of SKT with ASR and CC expressed a statistically significant activity to modulate the intestinal immune system ex vivo (Fig. 4). However, the F-3-related fraction from the formula of SKT alone did not show the activity as observed in the in vitro experiment. When each one single herb, excluding ASR, was removed from the set of five single herbs in the formula of SKT with ASR, and F-3related fractions were prepared from the decoctions of these modified combinations, none of the resulting F-3-related fractions showed the activity (Fig. 3B). This result strongly indicates that the combined decoction of five single herbs (Atractylodis Lanceae Rhizoma, Ginseng Radix, Poria, Glycyrrhizae Radix and ASR) is required for the expression of the modulatory activity on the intestinal immune system.

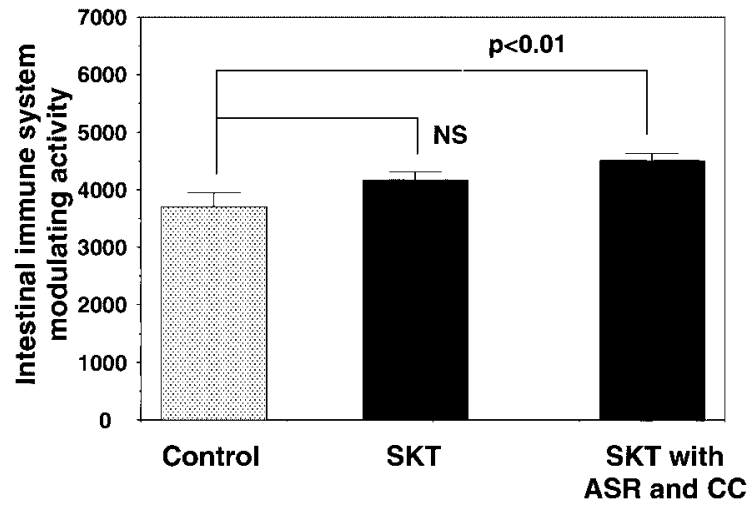

Figure 4. Ex vivo intestinal immune system modulating activity of F-3related fraction prepared from SKT or SKT with ASR and CC. 


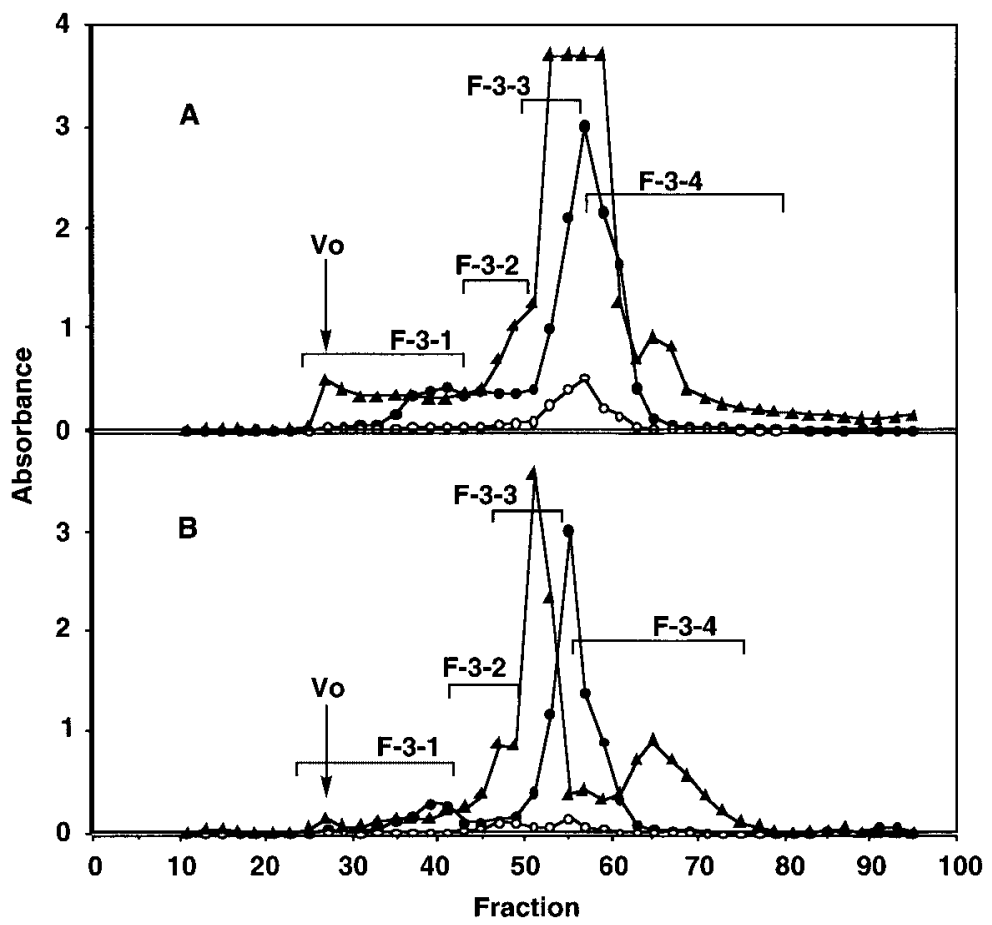

Figure 5. Gel filtration patterns on Sephadex G-25 of F-3-related fractions prepared from the formulation of SKT (A) or the combination of SKT with ASR and CC (B). Closed circles, carbohydrate $(490 \mathrm{~nm})$; open circles, uronic acid $(520 \mathrm{~nm})$; closed triangles, phenolics or protein $(280 \mathrm{~nm})$.

We subsequently performed the following experiment to comparatively analyze the difference of active components in the F-3-related fractions among different combinations of herbs. F-3-related fractions were first prepared by combined decoction of either the herbs of the SKT formula or SKT with ASR and CC, then fractionated by Sephadex G-25 gel filtration. Both of these F-3-related fractions gave small amounts of the highest-molecular-weight fraction (F-3-1), the intermediate size fraction (F-3-2), and large amounts of lower- and lowest-molecular-weight fractions (F-3-3 and F-34) (Fig. 5). All the subfractions from the F-3-related fraction of SKT with ASR and CC showed significantly higher intestinal immune system modulating activity than the corresponding subfractions from the F-3-related fraction of SKT (Fig. 6A). Among the subfractions from SKT with ASR and CC, F-3-1 showed the most potent activity in a dose-dependent manner (Fig. 6A and B), and F-3-4 had the second most potent activity, whereas F-3-2 and F-3-3 showed the weakest activities (Fig. 6A). When yields of these subfractions were compared, those of F-3-1 from SKT, and SKT with ASR and CC were similar, whereas the yield of F-3-4 from SKT with ASR and CC was almost twice as high as that of F-3-4 from SKT (Table 2). We considered that this result can explain one of the reasons why only the F-3-related fraction from SKT with ASR and CC showed the activity. It is also suggested that the combined decoction of the six single herbs from SKT with ASR and CC may increase the extraction efficiency of the active ingredients in F-3-4. It has been reported that the F-3-1-related fraction from TJ-48 comprises lignin-carbohydrate complexes as the active ingredients for the modulatory activity on the intestinal immune system (14). Carbohydrate contents of F-3-1 from SKT, and SKT with ASR and CC were similar, and component sugar analysis also indicated that both F-3-1 consisted mainly of glucose (Table 2). However, the lignin content of F-3-1 from SKT was remarkably higher than that from SKT with ASR and CC (Table 2). The presence of active and inactive lignin molecules has been suggested for modulation of the intestinal immune system (14), and the compositions of lignin-containing ingredients is assumed to be different between the F-3-1 subfraction from SKT and that from SKT with ASR and CC.

Components of F-3-4 from both multi-herbs seemed to be low molecular weight substances. In order to evaluate the difference of constituents between the subfractions, F-3-4 from SKT and SKT with ASR and CC, F-3-4 from both multiherbs were compared by 3D-HPLC. Because most of the ingredients in F-3-4 had maximal absorption near 250 and $280 \mathrm{~nm}$, elution profiles were analyzed by single wavelength at 250 or $280 \mathrm{~nm}$ (Fig. 7). Elution profiles of F-3-4 from SKT and that from SKT with ASR and CC were similar, with little differences in peak areas of constituents observed at $250 \mathrm{~nm}$. However, the areas of the peaks having retention times near 26.0 and 32.2 min were significantly higher in F-3-4 from SKT with ASR and CC than in F-3-4 from SKT in analyses at $280 \mathrm{~nm}$, and the peak having the retention time near 28.0 min in F-3-4 from SKT disappeared in that from SKT with ASR and CC. 


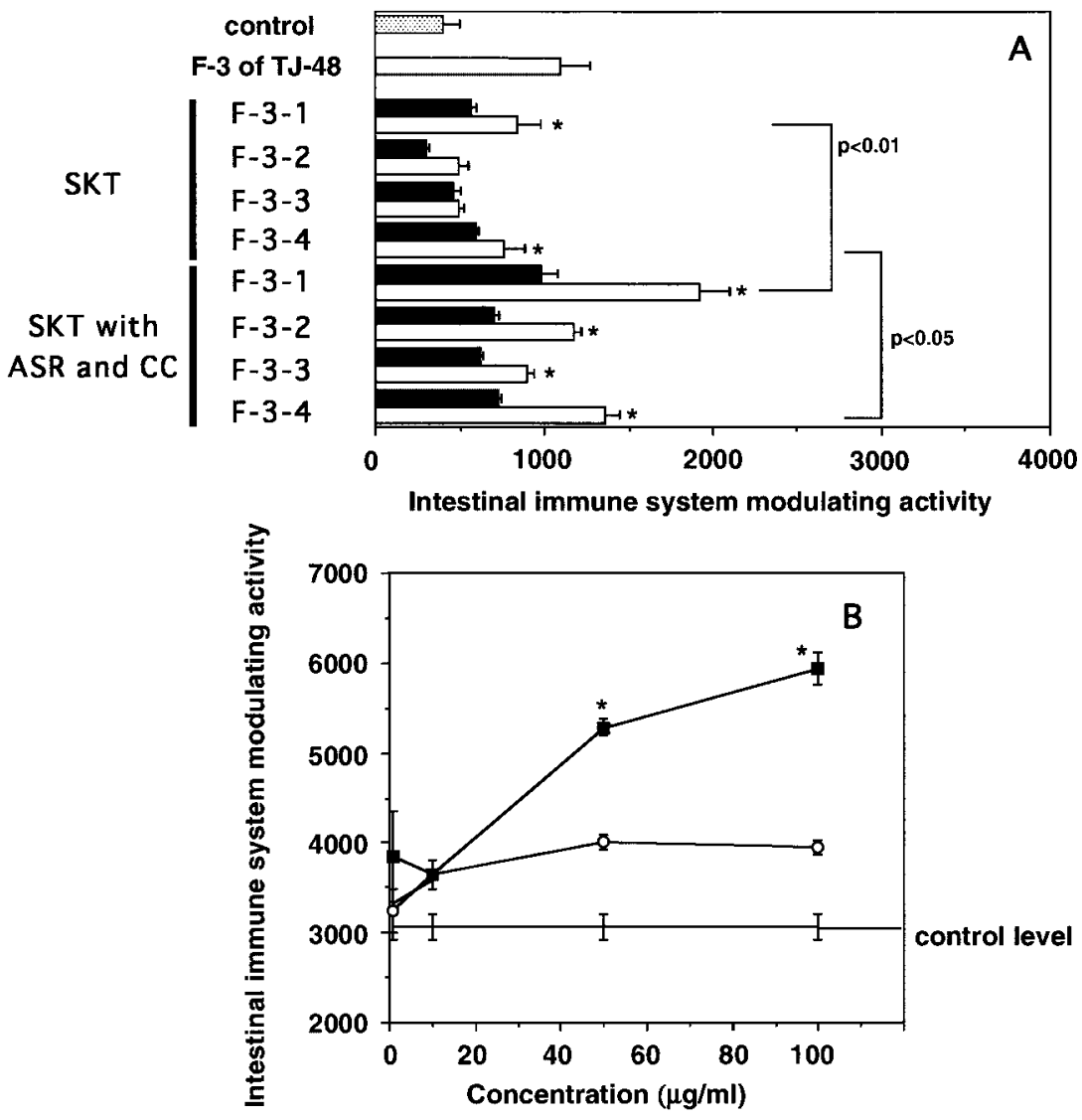

Figure 6. (A) Intestinal immune system modulating activity of subfractions of F-3-related fractions prepared from the formulation of SKT alone or from the combination of SKT with ASR and CC. Filled squares, $10 \mu \mathrm{g} / \mathrm{ml}$; open squares, $100 \mu \mathrm{g} / \mathrm{ml} .{ }^{*} P<0.05$ versus control. (B) Dose-dependent intestinal immune system modulating activity of F-3-1 from Fig. 5. Open circles, F-3-1 from SKT; filled squares, F-3-1 from SKT with ASR and CC. ${ }^{*} P<0.005$ versus data of F3-1 from SKT.

Table 2. Property of subfractions obtained from F-3 of the prescriptions of SKT, SKT with ASR and CC and TJ-48

\begin{tabular}{|c|c|c|c|c|c|c|c|c|c|}
\hline & \multicolumn{4}{|c|}{ SKT } & \multicolumn{4}{|c|}{ SKT with ASR and CC } & \multirow{2}{*}{$\begin{array}{l}\text { TJ-48 } \\
\text { F-3-1-1A }\end{array}$} \\
\hline & F-3-1 & F-3-2 & F-3-3 & F-3-4 & F-3-1 & F-3-2 & F-3-3 & F-3-4 & \\
\hline Yield $(\%)$ & 9.2 & 9.2 & 61.1 & 20.4 & 7.6 & 4.2 & 38.8 & 49.4 & \\
\hline Carbohydrate content $(\%)^{*}$ & 79.2 & & & & 84.9 & & & & n.d. \\
\hline Relative lignin content $(\%)^{\dagger}$ & 127.8 & & & & 57.0 & & & & 54.9 \\
\hline \multicolumn{10}{|l|}{ Component sugars (mol. \%) } \\
\hline Arabinose & 8.6 & & & & 9.0 & & & & 16.5 \\
\hline Rhamnose & 3.5 & & & & 2.9 & & & & 7.0 \\
\hline Fucose & 0.02 & & & & 0.06 & & & & 1.8 \\
\hline Xylose & 1.8 & & & & 2.1 & & & & 4.2 \\
\hline Glucuronic acid & 9.2 & & & & 3.6 & & & & 3.4 \\
\hline Galacturonic acid & 7.7 & & & & 4.8 & & & & 11.5 \\
\hline Mannose & 4.0 & & & & 8.0 & & & & 5.8 \\
\hline Galactose & 8.0 & & & & 6.0 & & & & 15.6 \\
\hline Glucose & 57.3 & & & & 63.2 & & & & 32.4 \\
\hline
\end{tabular}

*Glucose was used as standard.

†Commercially available lignin was used as standard.

Reported in Kiyohara et al. (14).

n.d., not determined. 


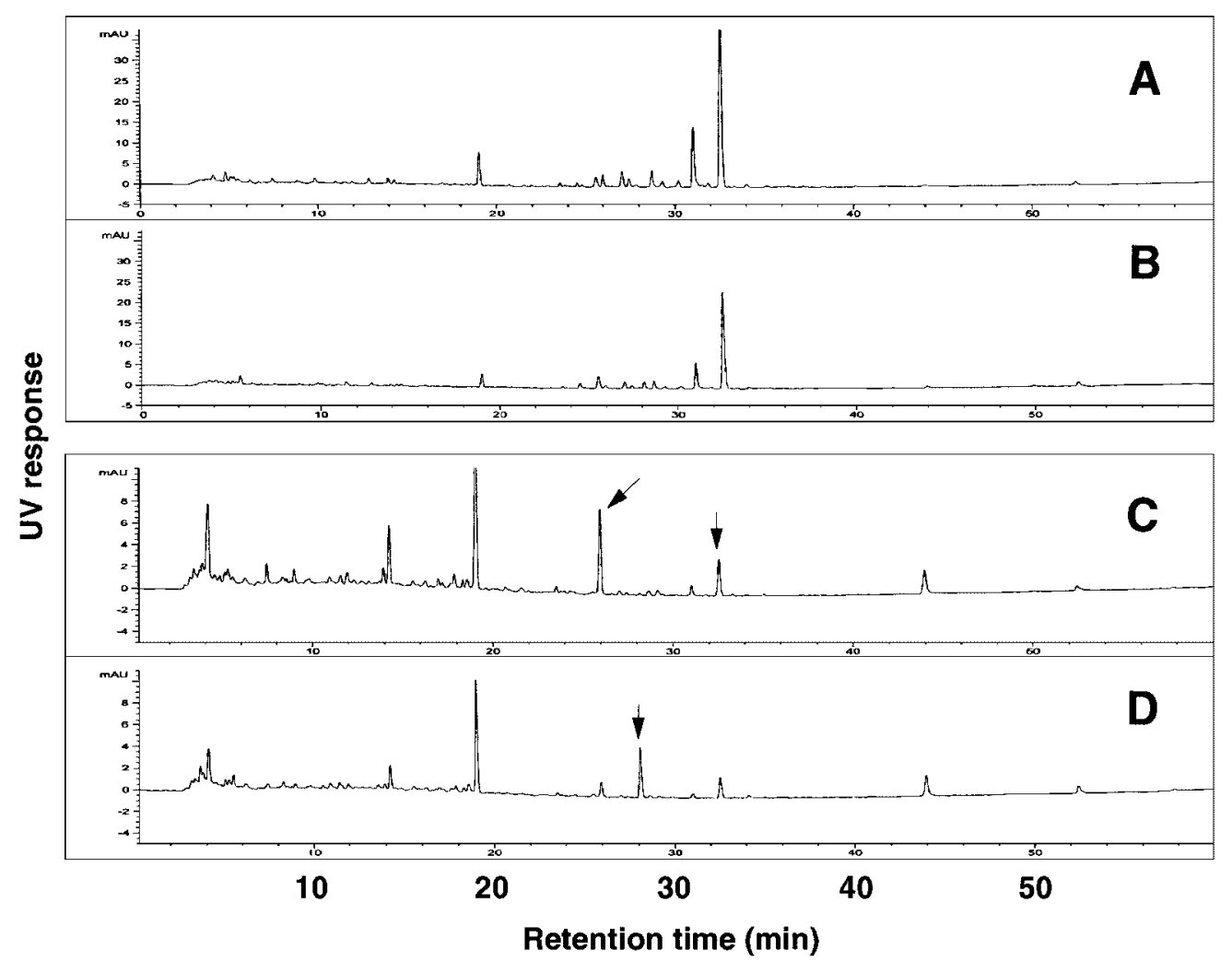

Figure 7. HPLC patterns of F-3-4 prepared from SKT with ASR and CC (A, C) and from SKT alone (B, D). (A, B) Monitored at $250 \mathrm{~nm}$; (C, D) Monitored at $280 \mathrm{~nm}$. The peaks with the arrows were significantly different between both F-3-4.

\section{Discussion}

In pharmacological study of the formulas in Kampo medicine, previous investigators have noted interesting phenomena when several single herbs are combined to be decocted into one formula. For example, Hosoya has indicated that antitussive action of the hot water extract of Ephedra Herba is enhanced by the combined decoction of this herb with three other kinds of single herbs, such as Armeniacae Semen, Glycyrrhizae Radix and Gypsum Fibrosum, while each of its hot water extracts alone has no antitussive action (1). It was also found that the duration and strength of antitussive effect of Ephedra Herba were prolonged and potentiated by this combined decoction compared with Ephedra Herba alone. As another example, a dried extract from one formula of Kampo medicine, Sho-saiko-to-go-Keishi-ka-shakuyaku-to (TJ-960), which consists of 10 kinds of single herbs, shows anti-convulsant action on seizures induced by the administration of pentetrazole to guinea pigs. However, if any single herb except Zingiberis Rhizoma from the multi-herbs of this formula is omitted, the anti-convulsant activity of the hot water extract from the complete formula disappears, suggesting that the combined decoction of nine single herbs in the formula of TJ-960 is necessary for expression of the activity (1). However, what constitutes the chemical, pharmacological and pharmaceutical basis for such phenomena of combination effects remains obscure.
The present results revealed that not all of the 10 single herbs in the formula of Juzen-taiho-to are required for the expression of its immuno-modulatory activity on the intestinal immune system. The combined decoction of six single herbs according to the formula of SKT (Atractylodis Lanceae Rhizoma, Ginseng Radix, Poria and Glycyrrhizae Radix) with ASR and CC is crucial for the activity of the F-3-related fraction to reach the same level as the F-3 of TJ-48. It is also suggested that the combined decoction of five single herbs (Atractylodis Lanceae Rhizoma, Ginseng Radix, Poria, Glycyrrhizae Radix, ASR) is essentially required for the expression of statistically significant activity. Although the F-3-related fraction prepared from the combined decoction of herbs due to SKT with ASR and CC also showed significant activity to modulate the intestinal immune system even in vivo, it was not as remarkable as that observed in the in vitro experiments. The experimental conditions such as dose of F-3-related fraction and period of oral administration need to be optimized in further study in order to evaluate the combination effect of the six kinds of single herbs due to SKT with ASR and CC in vivo.

The present study suggests that the SMT formula does not contribute to the expression of the activity, because its F-3related fraction after combined decoction did not show the activity at all, even if ASR or CC was further added to the multi-herbs of SMT. 
Juzen-taiho-to is known to have multifunctional properties in clinical applications. For example, Saiki et al. found that the combined decoction of the component herbs of SMT alone contribute to the expression of anti-metastatic activity of Juzen-taiho-to, but not that of the component herbs of SKT (19). Therefore, we suggest that SMT may play a different role from SKT for the expression of clinical effects of Juzen-taiho-to. The present study evaluated the effects of the combination of single herbs of Juzen-taiho-to only from the viewpoint of its modulating activity on the Peyer's patch cells in the intestinal immune system. We would therefore not exclude the possibility that the component herbs of SMT may play a role in modulation of the functions of other immune cells in the intestinal immune system.

The combined F-3-related fraction prepared by mixing of fractions containing lignin-carbohydrate complexes from 10 respective single herbs of Juzen-taiho-to failed to express the activity. This observation strongly suggests that the combined decoction of all essential herbs is quite important to bring about the combination of ingredients required for the expression of the activity. The present study indicates that yields of some low molecular weight ingredients were changed in decoction by the addition of ASR and CC to the multi-herbs of SKT. Therefore, we propose that the extraction efficiency of some active ingredients may be enhanced by the addition of ASR or ASR and CC to SKT.

The 3D-HPLC analysis clearly showed that some ingredients are either increased or disappear on the addition of ASR and CC to SKT; however, it is still not known whether the ingredients are decreased by the reaction with other ingredients in the herbs of SKT with ASR and CC. The highest molecular weight fraction, F-3-1, was assumed to contain lignin-carbohydrate complexes from the results of the study on the water-soluble and dialyzable fraction (F-3) of Juzentaiho-to for their modulatory activities on the intestinal immune system (14). The present results also allow us to postulate that even the relatively high molecular weight ingredients might also be changed in their content and/or structure by the combined decoction of multi-herbs. However, details of this change will require future clarification. The clarification must await further study on both high and low molecular weight ingredients regarding the precise events taking place on them during decoction of multi-herbs.

\section{Acknowledgments}

Part of the present work was supported by a fund from Tsumura \& Co., Tokyo. Part of the present work was also supported by 'The 21st Century COE Program' of the Ministry of Education, Culture, Sports, Science and Technology (MEXT) of Japan. We thank Ms F. Honma and Ms E. Yoshida for their technical assistance.

\section{References}

1. Hosoya E. Scientific reevaluation of Kampo prescriptions using modern technology. In Hosoya E, Yamamura Y. (eds) Resent Advances in the Pharmacology of Kampo (Japanese herbal) Medicines. Tokyo, Japan: Excerpta Medica 1988;17-29.

2. Komatsu Y, Takemoto N, Maruyama H, Tsuchiya H, Aburada M, Hosoya E, Shinohara S, Hamada H. Effects of Juzen-taiho-to on the anti-SRBC response in mice. Jpn J Inflamm 1986;6:405-8.

3. Maruyama H, Kawamura H, Takemoto N, Komatsu Y, Aburada M, Hosoya E. Effect of Juzen-taiho-to on phagocytes. Jpn J Inflamm 1988; $8: 461-5$

4. Takemoto N, Maruyama H, Kawamura H, Komatsu Y, Aburada M, Hosoya E. Mitogenic activity of Juzen-taiho-to (TJ-48) on murine lymphoid cells. Jpn J Inflamm 1989;9:137-40.

5. Takemoto N, Kiyohara H, Maruyama H, Komatsu Y, Yamada H, Kawamura H. A novel type of B-cell mitogen isolated from JuzenTaiho-To (TJ-48), a Japanese traditional medicine. Int J Immunopharmacol 1994;16:919-29.

6. Matsumoto T, Sakurai MH, Kiyohara H, Yamada H. Orally administered decoction of kampo (Japanese herbal) medicine, 'Juzen-Taiho-To' modulate cytokine secretion and induces NKT cells in mouse liver. Immunopharmacology 2000;46:149-61.

7. Kawamura H, Maruyama H, Takemoto N, Komatsu Y, Aburada M, Ikehara S, Hosoya E. Accelerating effect of Japanese kampo medicine on recovery of murine hemopoietic stem cells after administration of mitomycin C. Int J Immunother 1989;5:35-42.

8. Sugiyama K, Yokota M, Ueda H, Ichio Y. Protective effects of kampo medicines against cis-diaminedichloroplatinum (II)-induced nephrotoxicity and bone marrow toxicity in mice. J Med Pharm Soc Wakan-Yaku 1993;10:76-85.

9. Hisha H, Yamada H, Sakurai MH, Kiyohara H, Li Y, Yu CZ, et al. Isolation and identification of hematopoietic stem cell-stimulating substances from Kampo (Japanese herbal) medicine, Juzen-Taiho-To. Blood 1997;90:1022-30.

10. Hong T, Matsumoto T, Kiyohara H, Yamada H. Enhanced production of hematopoietic growth factors through $\mathrm{T}$ cell activation in Peyer's patches by oral administration of kampo (Japanese herbal) medicine, Juzen-Taiho-To. Phytomedicine 1998;5:353-60.

11. Kiyohara H. Elucidation of pharmacologically active polysaccharides from Kampo medicines and component herbs. In Ageta H, Aimi N, Ebizuka Y, Fujita T, Honda G. (eds) Towards Natural Medicine Research in the 21st Century. Tokyo, Japan: Elsevier 1998;161-71.

12. Yamada $\mathrm{H}$. The role of bioactive polysaccharides in Kampo medicines. In Watanabe H, Shibuya T. (eds) Pharmacological Research on Traditional Herbal Medicine. Amsterdam, the Netherlands: Harwood Academic Publishers 1999;179-96.

13. Yamada $\mathrm{H}$, Kiyohara $\mathrm{H}$. Complement-activating polysaccharides from medicinal herbs, In: Wagner H. (ed.) Immunomodulatory Agents from Plants. Basel, Switzerland: Birkhauser Verlag 1999;161-202.

14. Kiyohara H, Matsumoto T, Yamada H. Lignin-carbohydrate complexes: Intestinal immune system modulating ingredients in kampo (Japanese herbal) medicine, Juzen-Taiho-To. Planta Med 2000;66:204.

15. Kiyohara $\mathrm{H}$, Matsumoto $\mathrm{T}$. Yamada $\mathrm{H}$. Intestinal immune system modulating polysaccharides in a Japanese herbal (Kampo) medicine, Juzen-Taiho-To. Phytomedicine 2002;9:614-24.

16. Dubois M, Gilles KA, Hamilton JK, Rebers PA, Smith F. Colorimetric method for determination of sugars and related substances. Anal Chem 1956;28:350-6.

17. Blumenkrantz N, Asboe-Hansen G. New method for quantitative determination of uronic acid. Anal Biochem 1973;54:484-9.

18. Bradford MM. A rapid and sensitive method for the quantification of microgram quantities of protein utilizing the principle of protein-dye binding. Anal Biochem 1976;72:248-54.

19. Onishi Y, Yamaura T, Tauchi K, Sakamoto T, Tsukada K, Nunome S, Komatsu Y, Saiki I. Expression of the anti-metastatic effect induced by Juzen-taiho-to is based on the content of Shimotsu-to constituents. Biol Pharm Bull 1998;21:761-5. 


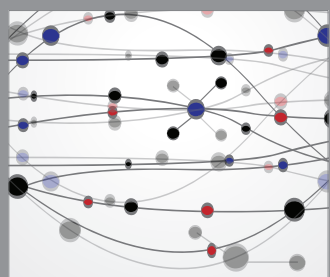

The Scientific World Journal
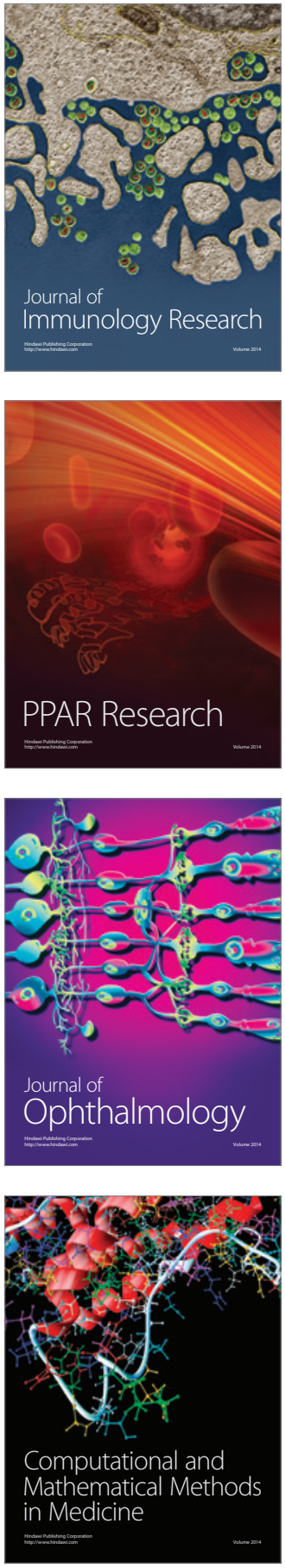

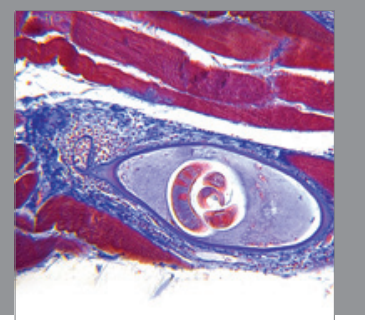

Gastroenterology

Research and Practice
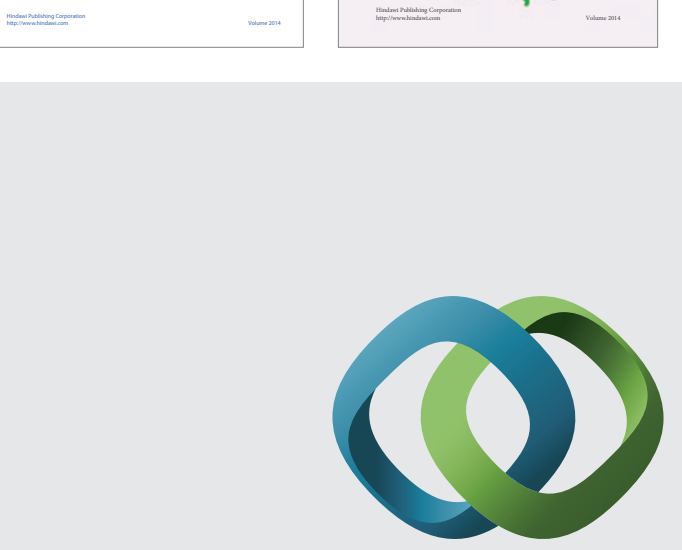

\section{Hindawi}

Submit your manuscripts at

http://www.hindawi.com
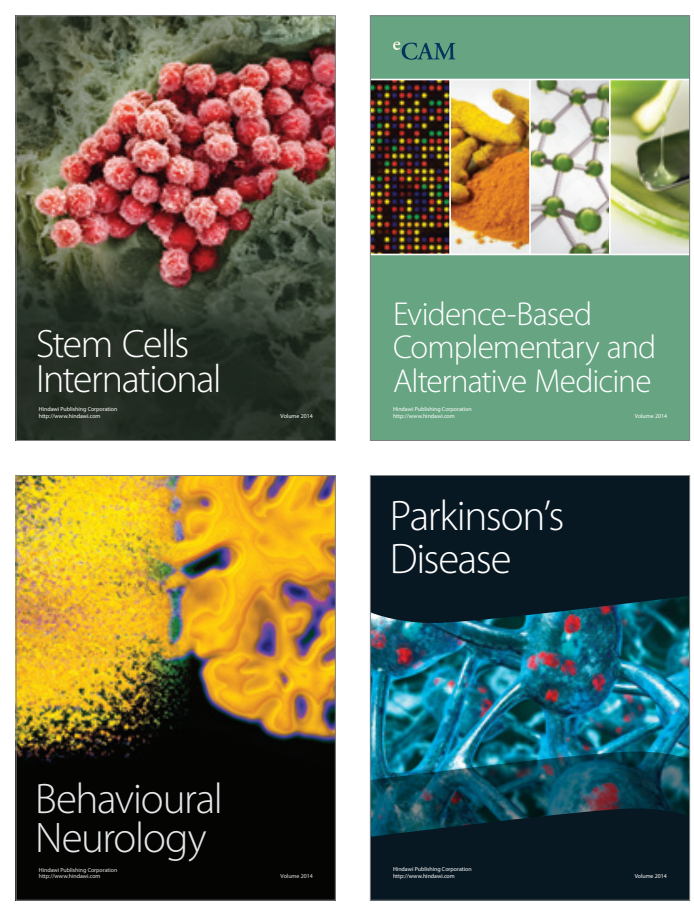

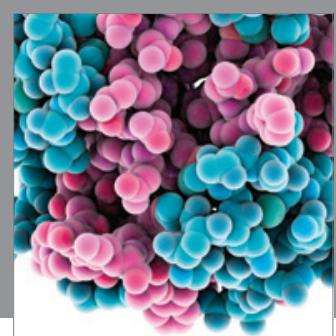

Journal of
Diabetes Research

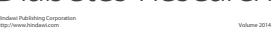

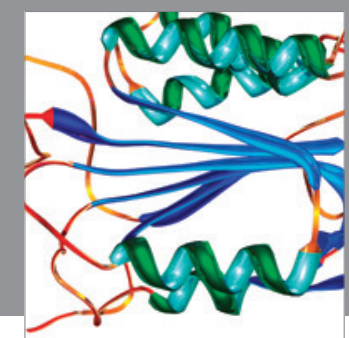

Disease Markers
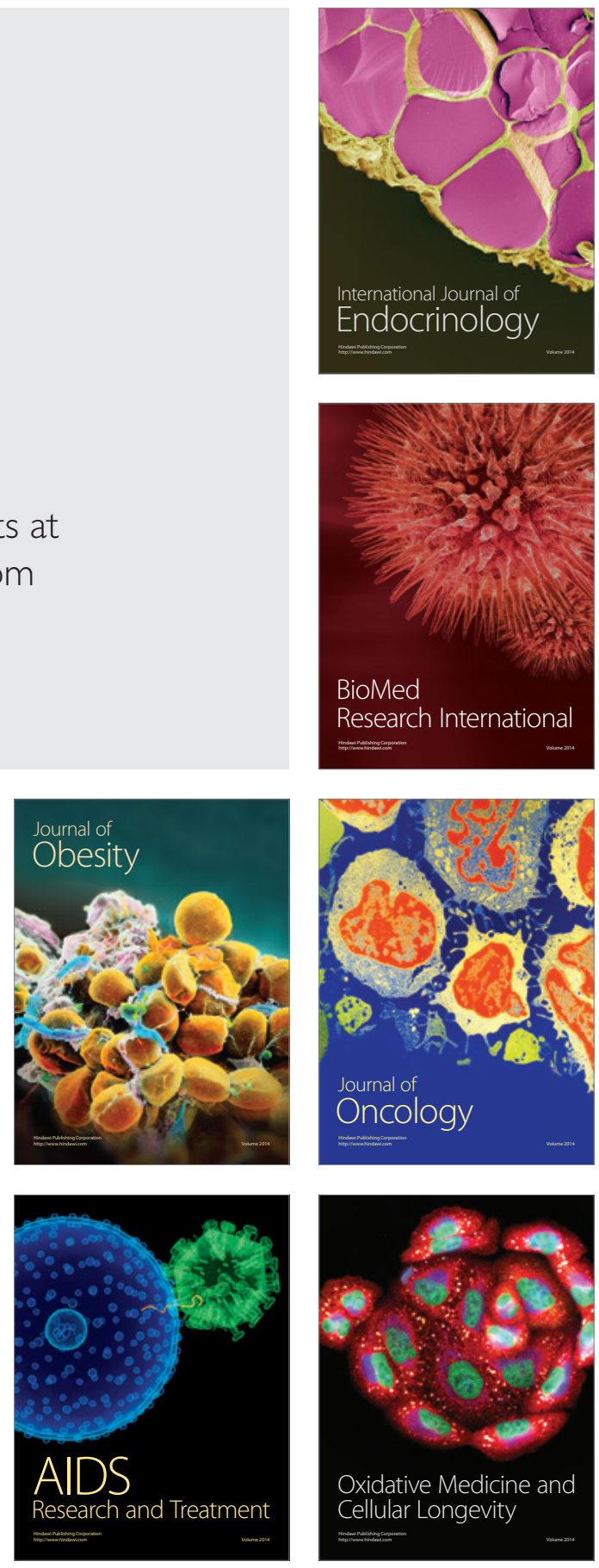